\title{
El potencial de la energía fotovoltaica como fuente de electricidad en Manabí
}

\section{The potential of photovoltaic energy as a source of electricity in Manabí}

1 Walter Iván Navas Bayona Universidad San Gregorio de Portoviejo winavas@sangregorio.edu.ec

2 Rayner Stalyn Durango Espinoza Escuela Superior Politécnica del Litoral rdurango@espol.edu.ec

3 Edgar Fabricio Landívar Chávez Investigador Independiente edgar@zensor.ec https://orcid.org/0000-0002-5646-1821

https://orcid.org/0000-0001-8162-1164

https://orcid.org/0000-0002-5464-4448

Artículo de Investigación Científica y Tecnológica

Enviado: 05/11/2021

Revisado: 20/11/2021

Aceptado: $27 / 12 / 2021$

Publicado:05/01/2022

DOI: $\underline{\text { https://doi.org/10.33262/cienciadigital.v6i1.1956 }}$

\section{Cítese:}

Navas Bayona, W. I., Durango Espinoza, R. S., \& Landívar Chávez, E. F. (2022). El potencial de la energía fotovoltaica como fuente de electricidad en Manabí. Ciencia Digital, 6(1), 91-115. https://doi.org/10.33262/cienciadigital.v6i1.1956

\footnotetext{
CIENCIA DIGITAL, es una Revista multidisciplinaria, Trimestral, que se publicará en soporte electrónico tiene como misión contribuir a la formación de profesionales competentes con visión humanística y crítica que sean capaces de exponer sus resultados investigativos y científicos en la misma medida que se promueva mediante su intervención cambios positivos en la sociedad. https://cienciadigital.org

La revista es editada por la Editorial Ciencia Digital (Editorial de prestigio registrada en la Cámara Ecuatoriana de Libro con No de Afiliación 663) $\underline{\text { www.celibro.org.ec }}$
}

Esta revista está protegida bajo una licencia Creative Commons AttributionNonCommercialNoDerivatives 4.0 International. Copia de la licencia: http://creativecommons.org/licenses/by-nc-nd/4.0/. 


\section{Palabras}

claves: cambio

climático,

energías

renovables,

radiación solar, energía

fotovoltaica.

\section{Keywords:}

Climate

change,

renewable

energies, solar radiation,

Photovoltaic

\section{Resumen}

Introducción. El cambio climático se mantiene como uno de los principales factores de riesgo para la humanidad, su efecto en las condiciones de vida y actividades de todos los seres vivos puede repercutir en forma drástica en el estilo de vida de los ecosistemas actuales. Diferentes países han implementado políticas y acciones que buscan mitigar el efecto de los gases invernadero y su contribución al cambio climático. El Ecuador ha implementado políticas para disminuir la emisión de gases contaminantes, modificando su matriz energética para aprovechar las condiciones climáticas y geográficas de la misma, buscando implementar el uso de energías renovables como la fotovoltaica. Objetivo. Analizar el potencial de generación eléctrica que se puede alcanzar en la provincia de Manabí, en el uso y aprovechamiento de la energía solar fotovoltaica. Metodología. Los datos se han obtenido por medio del uso de diferentes bases de datos de sistemas de energía solar, tomando valores de varias décadas referentes a la insolación solar, considerando como muestra los principales cantones de la provincia de Manabí; en donde se estimó los valores de generación de energía eléctrica que se puede alcanzar en cada cantón. Resultados. Los cantones de Manta y Montecristi alcanzan un alto nivel radiación solar superior a los $1,700 \mathrm{KWh} / \mathrm{m}^{2} / \mathrm{año}$; Portoviejo y Jipijapa alcanzan valor superior a $1,600 \mathrm{KWh} / \mathrm{m}^{2} / \mathrm{año}$, Chone y El Carmen valores ligeramente inferiores. Generando por metro cuadro con paneles de Silicio entre 205 a 262 KWh/año. Conclusión. La provincia de Manabí por su posición geográfica y sus condiciones climáticas muestra un alto potencial de desarrollo de energía de autoconsumo por medio de sistemas fotovoltaicos, lo cual permite plantear la viabilidad técnica de instalar sistemas fotovoltaicos a nivel de industrias, comercios y viviendas.

\section{Abstract}

Introduction. Climate change remains one of the main risk factors for humanity, its effect on the living conditions and activities of all living beings can drastically affect the lifestyle of current ecosystems. Different countries have implemented policies and actions that seek to mitigate the effect of greenhouse gases and their contribution to climate change. Ecuador has implemented policies to reduce the emission of polluting gases, 
modifying its energy matrix to take advantage of its climatic and geographical conditions, seeking to implement the use of renewable energies such as photovoltaics. Objective. Analyze the potential of electricity generation that can be achieved in the province of Manabí, in the use and exploitation of photovoltaic solar energy. Methodology. The data have been obtained using different databases of solar energy systems, taking values from several decades referring to solar insolation, considering as a sample the main cantons of the province of Manabí; where the electric power generation values that can be achieved in each canton were estimated. Results. The cantons of Manta and Montecristi reach a high level of solar radiation above 1,700 $\mathrm{KWh} / \mathrm{m}^{2} / \mathrm{year}$; Portoviejo and Jipijapa reach a value higher than 1,600 KWh/m²/year, Chone and El Carmen slightly lower values. Generating per square meter with Silicon panels between 205 to $262 \mathrm{KWh} /$ year Conclusion. Due to its geographical position and climatic conditions, the province of Manabí shows a high potential for the development of self-consumption energy through photovoltaic systems, which allows raising the technical feasibility of installing photovoltaic systems at the level of industries, businesses and homes.

\section{Introducción}

En las últimas décadas una serie de investigadores y organismos multilaterales han mencionado sobre la problemática global que implica el cambio climático para la humanidad. Convirtiéndose en el desafío y prioridad de organismos nacionales, internacionales, empresas, fundaciones y centros de investigación en todo el mundo. Siendo el tema principal de discusión y preocupación sus efectos en aspectos no solo ambientales, sino también en las dinámicas sociales, políticas y culturales (González \& Meira, 2020). La importancia de unir esfuerzos para investigar sobre los efectos del cambio climático está en las modificaciones de vida que puede generar esta problemática en las actividades humanas, cuando se considera que con las condiciones actuales el efecto puede ser irreversibles para el planeta (Schewe et al., 2019).

El esfuerzo para mitigar los efectos del cambio climático son múltiples, sin embargo su impacto se considera casi inevitable por cierto grupo de científicos, reconociendo que es un problema alarmante que requiere acciones inmediatas para evitar un avance de sus efectos negativos en el planeta (Canaza-Choque \& Huanca-Arohuanca, 2018). Esto ha 
permitido promover acciones que buscan retrasar sus efectos por medio de la adopción de estrategias y acciones que busquen mitigar la evidencia científica sobre esta problemática (Lewandowsky et al., 2015). Siendo la educación y socialización de los efectos del cambio climático una opción que los investigadores y grupos de interés manejan como política para difundir la importancia de concientizar a todos (Poma, 2018).

Sin embargo, la mitigación del cambio climático depende de la capacidad de la humanidad para controlar la emisión de los gases de efecto invernadero (GEI). Siendo esta la mayor dificultad actual de las naciones, porque las actividades antropogénicas es la causa principal del incremento de la temperatura del planeta y por ende de los cambios que se observa en varias regiones, países o lugares en las últimas décadas (Pérez \& Osal, 2018). En su gran mayoría estos gases son de orígenes naturales, pero principalmente se considera que se presentan por la quema de combustibles fósiles como el carbón y derivados del petróleo o inclusive por los procesos industriales que requieren para sus actividades, el consumo de sustancias que al liberarse contribuyen con GEI (Alcántara \& Padilla, 2005).

Como se ha mencionado, la dificultad del manejo en la emisión de gases de efecto invernadero, radica en las actividades humanas, en donde se considera que la agricultura, silvicultura y otros recursos contribuyen con la cuarta parte de las emisiones antropogénicas de GEI (IPCC, 2020). Otras acciones, son la generación de energía tomando como fuentes primarias al carbón y los derivados del petróleo, en donde la liberación de dióxido de carbono $\left(\mathrm{CO}_{2}\right)$ y monóxido de carbono $(\mathrm{CO})$ contribuyen al cambio de la atmosfera y al calentamiento global (Dong et al., 2019). En función de esta realidad, es que las naciones han desarrollado acciones conjuntas e individuales para mitigar la emisión de estos gases; de ahí que los objetivos de desarrollo del milenio (OMS) fue un gran avance para generar acciones y acuerdos para proteger al ambiente de las acciones del hombre, aunque no fueron suficiente (Reid, et al., 2017).

En el caso del Ecuador, el país ha suscrito y ratificado los objetivos de Desarrollo Sostenibles (ODS), que dentro de sus 17 objetivos y 169 metas contempla aspectos como justicia social, desarrollo económico, erradicación de la pobreza, igualdad y equidad, entro otros, así como al ambiente como una problemática mundial (Monteiro et al., 2019). Esta agenda global que plantea metas para el año 2030, se considera que aún tiene limitaciones de tipo técnico y político; debido al nivel de compromiso observado en ciertas naciones en la implementación de normativas ambientales internas que limiten la emisión de gases de efecto invernadero (Gil, 2018). A pesar de las críticas a las acciones emprendidas por varios países, el Ecuador mantiene una política de transformación de su matriz energética hacia fuentes de energía de tipo renovables, de ahí que la principal fuente de generación primaria es la hidroeléctrica. 


\section{Objetivos de Desarrollo Sostenible}

Los Objetivos de Desarrollo Sostenible (ODS) fueron adoptados por los países miembros de la Organización de las Naciones Unidas en el año 2015, con la aprobación de 193 países que firmaron la Resolución correspondiente. Estos ODS son la continuidad a lo planteado en los Objetivos de Desarrollo del Milenio (ODM), teniendo varios fines, que en su conjunto permitían mejorar las condiciones y calidad de vida en aspectos como la pobreza, seguridad alimentaria, protección del planeta, paz, prosperidad y otros; considerando como plazo para alcanzarlos el año 2030. Este nuevo compromiso se centra en aspectos críticos para la humanidad y el planeta, de forma solidaria y en su conjunto, es decir que las 169 metas deben ser alcanzadas por todos los países con la cooperación técnica, social y económica entre todas las naciones (Bórquez \& Lopicich , 2017).

Adicionalmente, en la resolución de la Asamblea General de las Naciones Unidas RES/70/1 del 2015, se aprecia el interés de los países por ejecutar acciones que beneficien a las personas, la paz universal, la prosperidad en todos sus ámbitos y ampliar el concepto de libertad; considerando esta resolución, que estos son los desafíos que debe enfrentar el mundo en los próximos años. Siendo importante mencionar como se considera el cuidado, manejo, protección y gestión del planeta:

Estamos decididos a proteger el planeta contra la degradación, incluso mediante el consumo y la producción sostenibles, la gestión sostenible de sus recursos naturales y medidas urgentes para hacer frente al cambio climático, de manera que pueda satisfacer las necesidades de las generaciones presentes y futuras (Asamblea General, 2015, p.2).

Un aspecto importante de los ODS, es que plantean una visión global, tratan de relacionar que el desarrollo de los países y de la sociedad en general es solo posible cuando aspectos como la desigualdad y la pobreza extrema sean atendidos y eliminados; que la sostenibilidad de la humanidad se da por medio de consumos sostenibles y con el manejo adecuado del ambiente; siendo necesario el desarrollo de capacidades técnicas, económicas y culturales para mitigar a niveles cero los problemas que generan desigualdades en las diferentes regiones (Gómez, 2018). Este planteamiento en los ODS de la intervención internacional es considerado como el pilar que genera esperanza sobre la posibilidad de alcanzar las metas planificadas o por lo menos lograr un alto porcentaje de cumplimiento de las metas; lo cual implicaría mejores condiciones de vida para todas las regiones.

De igual manera se observa que los informes de científicos e investigadores ratifican la preocupación por cumplir los ODS, en donde se menciona que los recursos naturales muestran signos de agotamiento, así como de degradación; que eleva los niveles de 
zonas o regiones con sequias y escasez de agua dulce; que en forma continua generan la pérdida de biodiversidad en muchas áreas del planeta (Ayuso, 2020). Siendo el Cambio Climático uno de los mayores retos de la humanidad por afectar la capacidad de las personas en alcanzar un desarrollo sostenible, dado el incremento de la temperatura global, que está generando la elevación del nivel de mar y acidez a los océanos; lo cual afecta a las zonas costeras y sus poblaciones, peligrando la supervivencia y modos de vida de muchos sistemas biológicos y vidas humanas.

Por esta preocupación hacia el ambiente y las consecuencias que las acciones humanas hacen en su entorno es que, dentro de los Objetivos de Desarrollo Sostenible, el ODS 7 se centra en "Garantizar el acceso a una energía asequible, segura, sostenible y moderna“ (Asamblea General, 2015, p.16). Este objetivo establece que un número significativo de la población mundial no tiene acceso a electricidad y que, a pesar del avance en la generación de energía, miles de millones de personas aún dependen de la madera, carbón y la biomasa para la cocción de alimentos o generación de energía térmica para hogares (Sachs et al., 2019).

Dentro de las acciones y estrategias para mantener el crecimiento energético como elemento de desarrollo económico de los países, está el uso de fuentes de energía renovables, siendo esta alternativa una de las principales políticas implementadas por varios grupos de interés alrededor del mundo. Este factor es esencial porque un gran porcentaje de las emisiones de gases de efecto invernadero son generadas por el consumo energético (Brandão et al, 2019). Por eso los países llevan varios años ajustando sus normativas legales nacionales para impulsar el uso de energías renovables, creando políticas de incentivos que permiten disminuir sus costos de instalación.

\section{Energías Renovables}

Las energías renovables durante las últimas décadas se han convertido en el eje central de desarrollo inicialmente de los objetivos de desarrollo del milenio (ODM) y ahora en una apuesta global en los Objetivos de Desarrollo Sostenible (ODS). En gran medida porque las fuentes de generación de energía por varios siglos fueron de fuentes contaminantes que emanan gases de efecto invernadero. El carbón, los derivados del petróleo y otros tipos de energía se convirtieron por muchas décadas en las fuentes de energía para movilidad, preparación de alimentos, procesos industriales y generación de energía eléctrica (Gielen et al., 2019). Existiendo la política de implementar una transición energética, por medio de la explotación de fuentes de generación primarias como el viento, sol, redes hidroeléctricas, geotermia y demás fuentes renovables que se consideran de difícil agotamiento y que su uso genera menor impacto en el ambiente que las fuentes energéticas tradicionales no renovables (Harjanne \& Korhonen., 2019). 
Estas políticas han permitido que las energías renovables muestren crecimientos constantes y sostenidos en los últimos años antes y después de la pandemia. Se estima que para fines del 2019 la generación de energía renovables alcanzó los 2,537.00 GW; en donde la energía hidroeléctrica alcanza la mayor representación con 1,190.00 GW; seguida de la energía eólica con 623.00 GW y la energía solar con 586.00 GW (IRENA, 2021). Sin embargo, se aprecia que otras fuentes de energía renovables son empleadas como mecanismos de generación como la bioenergía, la geotermia y la marina con valores de $638.00 \mathrm{GW}$. La figura 1 muestra el comportamiento de las energías renovables globales en los años previos a la pandemia.

\section{Figura 1}

\section{Energías renovables globales}

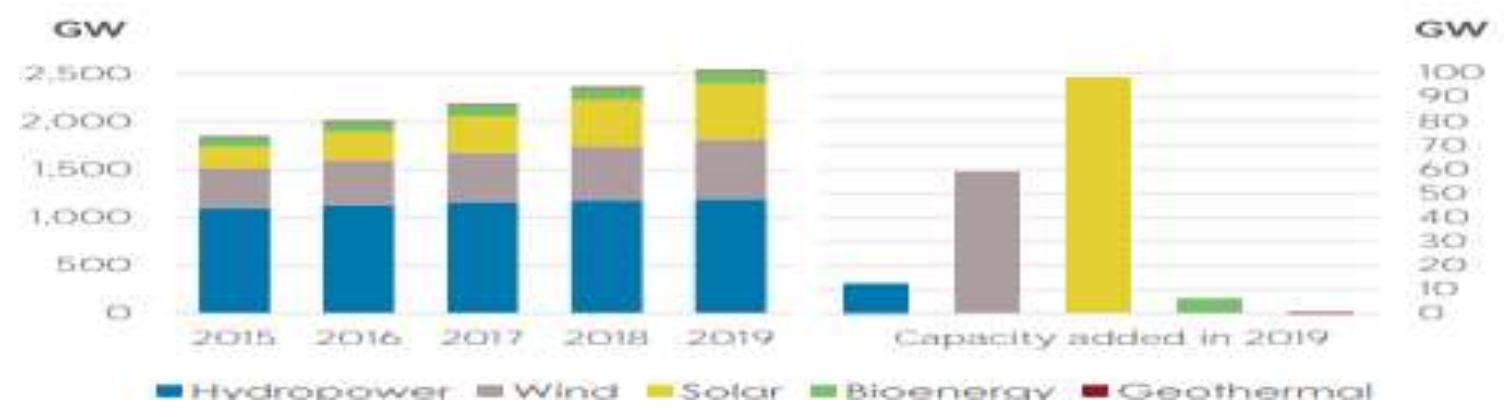

Fuente: Tomado de IRENA (2021). Renewable capacity statistics

De los valores del año 2019 se aprecia que en forma anual las energías renovables se incrementan en un $7.40 \%$, siendo la energía solar quien lidera el crecimiento con un $20.00 \%$ anual aproximadamente, seguida de la energía eólica con un $10.00 \%$ y la bioenergía con un $5.00 \%$. De igual manera, para el año 2020 se registra un estimado de crecimiento en la demanda energética del $10.30 \%$ anual, manteniéndose la energía solar como la de mayor crecimiento con un $22.00 \%$, seguida de la energía eólica con un $18.00 \%$. Se observa que el incremento del año 2020 obedece al desarrollo de fuentes energéticas en regiones como Asia, Norte América y Oceanía. Un factor importante es que en términos generales en los años 2019 y 2020 el sistema energético global mostró signos de tasas de bajo crecimiento, lo cual contribuyó al aumento porcentual de las energías renovables con respecto al total de energías.

Sin embargo, la importancia del análisis de las energías renovables es que se utiliza o emplea para producir electricidad; lo cual permite sustituir para la generación eléctrica a fuentes tradicionales como gas, carbón o combustibles (Wang et al., 2019). De los tipos de energía, la solar fotovoltaica se mantiene como la principal fuente de generación eléctrica en lo que respecta a crecimiento en los últimos años. En gran medida por la reducción de sus costos de instalación y una mejora gradual en la eficiencia de su tecnología, permitiendo que la solar fotovoltaica alcance valores muy competitivos por 
kWh en relación con todas las demás fuentes de energía. Bajo esta premisa, la energía solar fotovoltaica se considera como una alternativa energética que mantiene un crecimiento en todas las regiones y países; en gran medida por su alto desarrollo tecnológico, reducción de costos de producción y por la facilidad de acceso hacia esta fuente energética.

\section{Energía solar fotovoltaica}

La energía solar se produce por medio irradiación que hace una estrella, en nuestro sistema el sol es quien hace de fuente energética, emitiendo radiación solar que se propaga por medio de ondas electromagnéticas que generan un flujo en la atmosfera de $1,367.00 \mathrm{~W} / \mathrm{m}^{2}$, variando a lo largo del año entre 1,321.00 a 1,412.00 W/m². Al ingresar a la atmosfera esta radiación es sometida a diferentes procesos que modifican sus características, estos cambios se dan por efecto de las nubes, vapor de agua, ozono y el dióxido de carbono (Perpiñán, 2013). Esta incidencia de la energía solar se aprovecha para varios fines, en forma pasiva sin dispositivos de captación y en forma activa con dispositivos de captación. En los sistemas activos se puede aprovechar la radiación solar para generar energía solar térmica, la cual aprovecha la temperatura por medio de colectores solares para generar energía térmica, o energía fotovoltaica que aprovecha la luz solar para convertirle en energía eléctrica.

La energía solar fotovoltaica es el proceso más empleado para el aprovechamiento de la energía solar; esta se centra en el principio del efecto fotoeléctrico para generar electricidad. Este principio menciona que las ondas electromagnéticas solares están compuestas por fotones que son partículas indivisibles y que se consideran como un elemento fundamental; permitiendo esto definir a este tipo de energía como renovable, porque la radiación solar es inagotable y no contaminante (García et al., 2021). Para la conversión de la energía, el sistema requiere de un dispositivo de células solares encapsuladas en módulos solares, que con la electrónica e interconexión desarrollan un principio de semiconductores. El fotón al incidir en el módulo solar ilumina al semiconductor el mismo que absorbe dicha energía, que le permite liberar electrones para que puedan saltar de la banda de valencia a la de conducción.

Por lo sencillo de su principio y por el aprovechamiento del recurso solar, el mercado de energías fotovoltaicas mantiene un crecimiento constante por encima del promedio de otras fuentes de energías renovables. Su crecimiento se centra en ocho mercados o países que en forma continua impulsan el crecimiento de este tipo de energía. Para el año 2020, a pesar de que el valor cambia de acuerdo con la fuente, se estima que se instalaron $93.00 \mathrm{GW}$, valor mayor a los $69.00 \mathrm{GW}$ del año 2019; siendo los principales actores China con $48.00 \mathrm{GW}$, Estados Unidos con $15.00 \mathrm{GW}$ y Vietnam con $8.00 \mathrm{GW}$ (Feldman \& Margolis, 2021). La figura 2 muestra la variación interanual del mercado fotovoltaico, en donde los ocho principales mercados concentran aproximadamente el 
$75 \%$ de las instalaciones a nivel mundial; mostrando la alta contribución gradual que concentra esta fuente de energía que en su gran mayoría es empleada para generación de electricidad.

\section{Figura 2}

\section{Incremento anual de energía fotovoltaica}

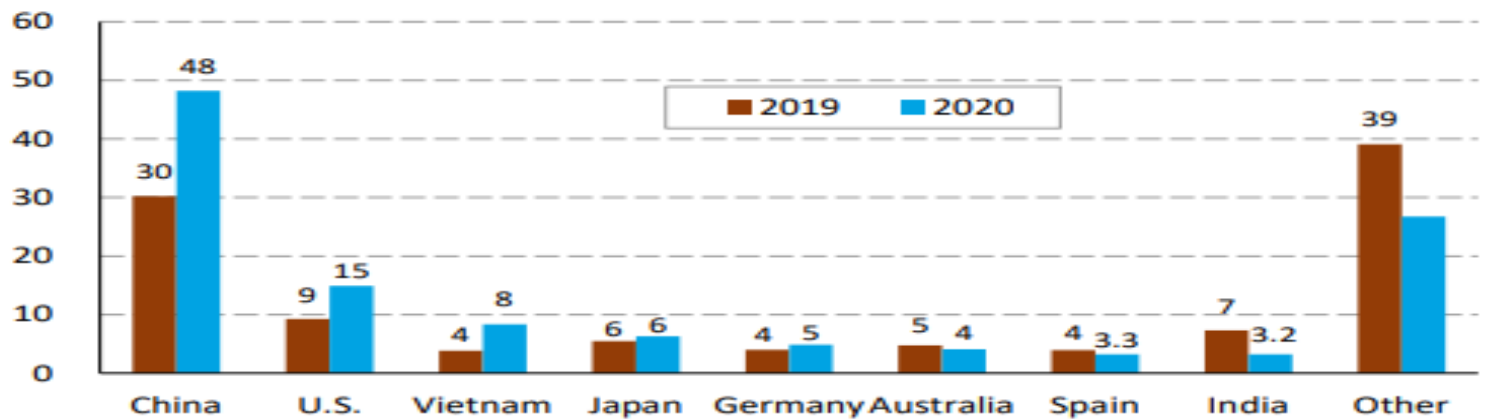

Fuente: Tomado de Feldman et al. (2021). Solar Industry Update

Como se ha mencionado el desarrollo de la energía fotovoltaica se da por el avance tecnológico que se observa en sus equipos. Siendo el que más contribuye a facilitar la instalación por su desarrollo los paneles fotovoltaicos. Mostrando la literatura un amplio espectro de elementos utilizados para construir los mismos, siendo los de silicio cristalino los que han dominado el mercado; sean de tipo monocristalinos o policristalinos, porque el silicio es un elemento muy abundante en el planeta. A pesar de que los paneles de silicio son los de mayor uso comercial, existen otros elementos con mayor rendimiento alcanzado en laboratorios, como las placas compuestas de a-Si, CdTe, CIS, CIGS, de amorfos de capa fina, entre otros. Mostrando la Figura 3 la eficiencia de las diferentes tecnologías, en donde se observa rendimientos experimentales de placas que alcanzan más de un $40 \%$ de eficiencia.

\section{Figura 3}

\section{Eficiencia de módulos fotovoltaicos}

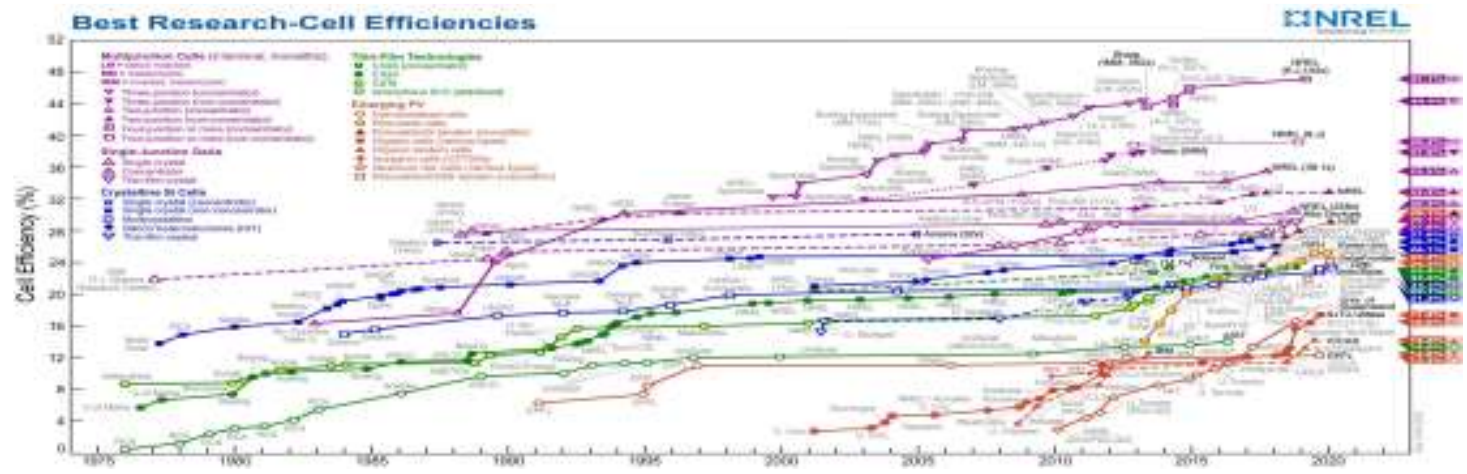

Fuente: Tomado de Feldman \& Margolis. (2021). H2 2020: Solar Industry Update 
Esta alta eficiencia alcanzada por ciertas tecnologías en los paneles solares, no logran crear condiciones suficientes que permitan modificar la demanda global sobre paneles de silicio, los mismo que llevan años manteniéndose como los de mayor viabilidad comercial por el efecto combinado de costo-rendimiento que alcanzan en las instalaciones que emplean estos equipos. Apreciándose que en el caso de los módulos fotovoltaicos mono c-Si aumentaron un $6 \%$ en el primer trimestre de 2021, siendo ligeramente más altos que hace un año, mientras que los de policristalinos de silicio se incrementaron en el primer trimestre del 2021 en un 33\% a nivel mundial (Feldman \& Margolis, 2021).

\section{Energía Eléctrica y fotovoltaica en Ecuador}

La electricidad es un recurso esencial para el desarrollo y progreso de las sociedades en todas las regiones y países actualmente, existen estudios que mencionan que el crecimiento económico y financiero de las economías nacionales están asociadas con el incremento en el consumo de energía eléctrica y otras fuentes energéticas (Caraballo \& García-Simón, 2017). En función de este supuesto, los países consideran al sector eléctrico como área estratégica para el funcionamiento de sus sociedades y de las economías, generando reglamentaciones y leyes que buscan crear las condiciones adecuadas de inversión, generación, comercialización y control.

Para el caso del Ecuador en el año 2018 la capacidad instalada de generación a nivel nacional registró los 8,676.89 MW de potencia nominal y 8.062,58 MW de potencia efectiva; en donde las energías renovables registran los 5,271.74 MW de generación, lo que implica un $60,75 \%$ del total del sistema; mientras que las energías no renovables generan 3,405.14 MW que implican el 39,24 \% del total del sistema (Agencia de Regulación y Control de Electricidad [ARCONEL], 2019). Dentro del sistema de generación eléctrica del país, la energía hidroeléctrica es la fuente primaria de mayor aporte, alcanzando los 5,071.40 MW de potencia nominal, seguida de la generación térmica por medio de motores de combustión interna con 2,021.43 MW de potencia nominal y en tercer lugar la térmica con gas con 921,85 MW de potencia nominal. La Energía fotovoltaica alcanza una contribución mínima con un 27,63 MW, mostrando un leve incremento en relación con años anteriores; la misma que está distribuida en proyectos energéticos situados en 10 provincias del país, en donde las provincias de Loja y El Oro alcanzan proyectos que en su conjunto registra los $6 \mathrm{MW}$ de potencia nominal.

Dada la importancia del incremento en la capacidad instalada del sistema eléctrico del país, y en función de las normativas vigentes, las políticas nacionales vigentes buscan impulsar el uso de las energías renovables como fuentes de generación energética. Existiendo desde hace años diferentes planes de acción que buscan cambiar la matriz energética del país, creando condiciones e incentivos para la implementación de energía 
renovables. Debido a esta planificación, el Consejo Nacional de Electricidad (CONELEC) presentó en el año 2008 el Atlas Solar del Ecuador para Fines de Generación Eléctrica; documento que fue generado por la Nacional Renewable Energy Laboratory (NREL) que ejecuta acciones de investigación y desarrollo de energías renovables y eficiencia energética.

Dentro de este marco NREL implementó su modelo Climatological Solar Radiation Model, para conocer la radiación solar diaria en superficies horizontales en celdas; sistema que ha sido empleado en todo el planeta y que toma datos de estaciones con un error estimado del 10\%. La información contenida en el Atlas Solar del año 2008 tomó datos desde el año 1985 hasta el 2006. Los resultados indican que en promedio en el país la insolación global alcanza los 4,574.99 $\mathrm{Wh} / \mathrm{m}^{2} /$ día, con valores máximos de $5,748.00 \mathrm{Wh} / \mathrm{m}^{2} /$ día y valores mínimos de 3,634.00 Wh/ $/ 2 /$ día; en donde se aprecia provincias con alto potencial como Loja, Santo Domingo, Pichincha e Imbabura. En el caso de Manabí se aprecian valores entre 4,350.00 Wh/m²/día a 5,200.00 Wh/m²/día; mostrando el nivel de insolación global que incide en la provincia.

Sin embargo, existe una limitada información sobre la irradiación solar potencial en varios cantones o puntos de Manabí, la cual puede determinar las condiciones potenciales de la energía fotovoltaica que puede ser aprovechada en la provincia como opción para la generación de electricidad (Vera et al., 2017). El presente trabajo busca analizar el potencial energético solar fotovoltaico de la provincia de Manabí para la generación de energía eléctrica de fuentes renovables, que contribuya a minimizar el impacto de los gases de efecto invernadero y con ello contribuir en aminorar el efecto del cambio climático.

\section{Metodología}

La investigación se centra en un enfoque de investigación cuantitativa de tipo longitudinal, por medio de la revisión de series históricas de información sobre la irradiación solar en diferentes ciudades de la provincia de Manabí, buscando determinar el valor potencial de la provincia para la generación de electricidad. Para este propósito se realizará estudios de casos que permitan generar un estudio descriptivo sobre las características de la irradiación solar y el valor que se puede aprovechar como fuente energética. Se considera como muestra los seis cantones de la provincia con mayor número de habitantes, tomando como base el último censo de población y vivienda del año 2010 y las proyecciones de crecimiento poblacional de los mismos; como se muestra en la tabla 1. 
Tabla 1

Población de los principales cantones de Manabí

\begin{tabular}{ccc}
\hline CANTONES & $\mathbf{2 0 1 0}$ & $\mathbf{2 0 2 0}$ \\
\hline PORTOVIEJO & $290,199.00$ & $321,800.00$ \\
MANTA & $234,547.00$ & $264,281.00$ \\
CHONE & $131,674.00$ & $131,002.00$ \\
EL CARMEN & $91,918.00$ & $111,344.00$ \\
JIPIJAPA & $73,951.00$ & $74,645.00$ \\
MONTECRISTI & $71,947.00$ & $107,785.00$
\end{tabular}

Fuente: Adaptado de Instituto Nacional de Estadísticas y Censos (INEC, 2010)

Definidas las ciudades de la provincia de Manabí consideradas para el estudio, se situó en cada cantón un punto de referencia donde ejecutar la simulación de los cálculos de irradiación solar. Para este propósito y debido a las diferentes opciones, se estimó tomar como puntos de referencia los edificios de las Municipalidades de cada cantón, lugares que pueden ser considerados como referentes de zonas urbanas. Para este propósito en la tabla 2 se aprecia las coordenadas de cada Municipio por cantón.

\section{Tabla 2}

Población de los principales cantones de Manabí

\begin{tabular}{lllll}
\hline CANTONES & \multicolumn{2}{l}{ LATITUD } & \multicolumn{2}{l}{ LONGITUD } \\
\hline PORTOVIEJO & - & 1.05790 & - & 80.44990 \\
MANTA & - & 0.94840 & - & 80.72150 \\
CHONE & - & 0.69840 & - & 80.09440 \\
EL CARMEN & - & 0.27190 & - & 79.45170 \\
JIPIJAPA & - & 1.34810 & - & 80.58000 \\
MONTECRISTI & - & 1.05020 & - & 80.66030 \\
\hline
\end{tabular}

Fuente: Google map

Para la estimación de la irradiación solar se procedió a contrastar la información emitida por el CONELEC en el Atlas Solar del Ecuador para Fines de Generación Eléctrica, para este propósito se ha tomado varias fuentes de estimación de energía solar como METEONEORM que es un software desarrollado en Suiza y que tiene varias versiones, en donde se ha tomado la que estima la radiación solar con datos desde el 2010 al 2014. Otra fuente que se consideró es la Base de Datos Nacional de Radiación Solar (NSRDB) del software de NREL que emplea mediciones multicanal de satélites geoestacionarios, cuyo periodo considerado para esta investigación contempla desde 1998 a 2016. Otra base de datos empleada fue la del Sistema de Información Geográfica 
Fotovoltaica (PVGIS), que es desarrollada por el Centro Común de Investigación de la Comisión Europea, la cual toma rango entre los años 2005 a 2016.

\section{Resultados}

Las estimaciones de radiación solar con las bases de datos de los cuatro programas en los seis cantones indican que en el caso de Portoviejo el valor anual de radiación esta entre $1,546.00 \mathrm{kWh} / \mathrm{m}^{2} /$ año y $1,759.00 \mathrm{kWh} / \mathrm{m}^{2} /$ año (tabla 3 ); valores que muestran una diferencia significativa que se produce por la base de datos utilizada, el proceso de captación de información o por los años que se toman como muestra. Pero que permiten apreciar que hay un potencial solar que puede aprovecharse para generación de electricidad.

Tabla 3

Irradiación Solar en Portoviejo ( $\left.\mathrm{kWh} / \mathrm{m}^{2} / \mathrm{mes}\right)$

\begin{tabular}{lcccc}
\hline MES & METEONEORM & NASA & PVGIS TMY-SARAH & NREL NSRDB \\
\hline ENE & 135 & 170 & 112 & 116 \\
FEB & 128 & 148 & 110 & 117 \\
MAR & 158 & 180 & 140 & 151 \\
ABR & 163 & 172 & 151 & 143 \\
MAY & 153 & 162 & 132 & 130 \\
JUN & 116 & 123 & 102 & 117 \\
JUL & 124 & 114 & 102 & 121 \\
AGO & 137 & 124 & 133 & 134 \\
SEP & 128 & 131 & 141 & 153 \\
OCT & 104 & 136 & 155 & 138 \\
NOV & 115 & 138 & 131 & 152 \\
DIC & 138 & 162 & 136 & 135 \\
\hline ANUAL & $1,598.00$ & $1,759.00$ & $1,546.00$ & $1,608.00$
\end{tabular}

Para el caso de Manta ciudad que cuenta con puntos de medición local de irradiación solar, los valores de las bases de datos están entre $1,651.00 \mathrm{kWh} / \mathrm{m}^{2} /$ año a $1,874.00$ $\mathrm{kWh} / \mathrm{m}^{2} / \mathrm{año}$ (tabla 4); lo cuales indican y muestran el potencial energético de la ciudad y explica porque hay proyectos de plantas solares en la misma a nivel comercial. Adicionalmente la ciudad de Manta cuenta con varias industrias que empiezan a ver a este tipo de energía como una opción viable. 


\section{Tabla 4}

Irradiación Solar en Manta $\left(\mathrm{kWh} / \mathrm{m}^{2} / \mathrm{mes}\right)$

\begin{tabular}{lcccc}
\hline MES & METEONEORM & NASA & PVGIS TMY-SARAH & NREL NSRDB \\
\hline ENE & 148 & 164 & 138 & 161 \\
FEB & 137 & 147 & 142 & 140 \\
MAR & 167 & 184 & 163 & 177 \\
ABR & 172 & 174 & 167 & 171 \\
MAY & 160 & 163 & 156 & 152 \\
JUN & 115 & 122 & 143 & 136 \\
JUL & 120 & 124 & 150 & 151 \\
AGO & 130 & 131 & 159 & 155 \\
SEP & 124 & 135 & 154 & 158 \\
OCT & 103 & 141 & 164 & 158 \\
NOV & 122 & 139 & 156 & 158 \\
DIC & 152 & 157 & 169 & 159 \\
\hline ANUAL & 1,651 & 1,780 & 1,862 & 1,874
\end{tabular}

Para el caso de Chone que se ubica en el norte de la provincia y que sus valores son estimados en función de datos de equipos de medición cercanos, los valores de irradiación anual están entre $1,546.00 \mathrm{kWh} / \mathrm{m}^{2} /$ año y $1,759.00 \mathrm{kWh} / \mathrm{m}^{2} /$ año (tabla 5 ). Esto indica un potencial solar para los sectores económicos del cantón como el sector agrícola y pecuario, los cuales cuentan con sistemas de generación de electricidad por medio fotovoltaicos cuyos equipos son aislados, es decir no conectados a la red por la dificultad de estos sectores de tener acceso a la red pública.

\section{Tabla 5}

Irradiación Solar en Chone $\left(\mathrm{kWh} / \mathrm{m}^{2} / \mathrm{mes}\right)$

\begin{tabular}{lcccc}
\hline MES & METEONEORM & NASA & PVGIS TMY-SARAH & NREL NSRDB \\
\hline ENE & 140 & 164 & 103 & 123 \\
FEB & 131 & 147 & 139 & 126 \\
MAR & 157 & 184 & 151 & 162 \\
ABR & 163 & 174 & 145 & 146 \\
MAY & 150 & 163 & 129 & 133 \\
JUN & 109 & 122 & 106 & 111 \\
JUL & 113 & 124 & 108 & 114 \\
AGO & 123 & 131 & 112 & 125 \\
SEP & 115 & 135 & 116 & 131 \\
\hline
\end{tabular}


Tabla 5

Irradiación Solar en Chone ( $\left.\mathrm{kWh} / \mathrm{m}^{2} / \mathrm{mes}\right)$ (continuación)

\begin{tabular}{lcccc}
\hline MES & METEONEORM & NASA & PVGIS TMY-SARAH & NREL NSRDB \\
\hline OCT & 93 & 141 & 137 & 129 \\
NOV & 113 & 139 & 128 & 122 \\
DIC & 139 & 157 & 133 & 123 \\
\hline ANUAL & 1,546 & 1,780 & 1,506 & 1,545
\end{tabular}

Para el caso del cantón El Carmen que se ubica en el norte de la provincia y que se caracteriza por su sector pecuario y agrícola como principales fuentes de generación económica, los valores de irradiación anual están entre $1,296.00 \mathrm{kWh} / \mathrm{m}^{2} / \mathrm{año} \mathrm{y}$ 1,478.00 $\mathrm{kWh} / \mathrm{m}^{2} /$ año (tabla 6); los mismos que indica un nivel de radiación solar aceptable, que tiene un potencial para la generación de electricidad, pero que alcanzaría viabilidad económica bajo ciertas condiciones. Siendo importante mencionar que en este cantón las conexiones fuera de la red, se contempla como una opción para el sector agropecuario que tiene dificultad de acceso a la red pública.

\section{Tabla 6}

Irradiación Solar en El Carmen $\left(\mathrm{kWh} / \mathrm{m}^{2} / \mathrm{mes}\right)$

\begin{tabular}{lcccc}
\hline MES & METEONEORM & NASA & PVGIS TMY-SARAH & NREL NSRDB \\
\hline ENE & 131 & 123 & 95 & 117 \\
FEB & 121 & 120 & 121 & 117 \\
MAR & 140 & 147 & 144 & 142 \\
ABR & 149 & 135 & 109 & 126 \\
MAY & 145 & 122 & 116 & 113 \\
JUN & 118 & 107 & 96 & 98 \\
JUL & 121 & 112 & 111 & 102 \\
AGO & 128 & 125 & 98 & 111 \\
SEP & 116 & 123 & 105 & 117 \\
OCT & 95 & 118 & 106 & 112 \\
NOV & 97 & 113 & 95 & 108 \\
DIC & 118 & 117 & 100 & 109 \\
\hline ANUAL & 1,478 & 1,462 & 1,296 & 1,369
\end{tabular}

El cantón Jipijapa que posee características geográficas particulares con continuas elevaciones a lo largo de superficie, registra los valores de irradiación anual que están entre $1,520.00 \mathrm{kWh} / \mathrm{m}^{2} /$ año y $1,759.00 \mathrm{kWh} / \mathrm{m}^{2} /$ año (tabla 7 ); debiendo mencionarse que en este cantón hay empresarios del sector agropecuario que por las dificultades de 


\section{www.cienciadigital.org}

cobertura de la red pública de electricidad cuentan con sistemas de generación fotovoltaicas como opción para el encendido de motores o alumbrado de haciendas.

\section{Tabla 7}

Irradiación Solar en Jipijapa $\left(\mathrm{kWh} / \mathrm{m}^{2} / \mathrm{mes}\right)$

\begin{tabular}{lcccc}
\hline MES & METEONEORM & NASA & PVGIS TMY-SARAH & NREL NSRDB \\
\hline ENE & 130 & 170 & 102 & 110 \\
FEB & 124 & 148 & 107 & 112 \\
MAR & 154 & 180 & 133 & 143 \\
ABR & 159 & 172 & 135 & 130 \\
MAY & 152 & 162 & 115 & 125 \\
JUN & 123 & 123 & 97 & 113 \\
JUL & 131 & 114 & 126 & 124 \\
AGO & 143 & 124 & 129 & 142 \\
SEP & 134 & 131 & 161 & 154 \\
OCT & 112 & 136 & 160 & 150 \\
NOV & 108 & 138 & 131 & 139 \\
DIC & 132 & 162 & 124 & 135 \\
\hline ANUAL & 1,603 & 1,759 & 1,520 & 1,576
\end{tabular}

El cantón Montecristi que se ubica entre Portoviejo y Manta, que su economía se desarrolla con varios sectores, lo cual permite una amplitud de ingresos para sus habitantes, inclusive porque se han desarrollado proyectos inmobiliarios en los últimos años y se han instalada nuevas industrias en su zona industrial, registra valores entre $1,630.00 \mathrm{kWh} / \mathrm{m}^{2} /$ año y $1,759.00 \mathrm{kWh} / \mathrm{m}^{2} /$ año (tabla 8). Siendo importante mencionar que por las actividades económicas se observa en este cantón instalaciones de micro generación en pocas industrias y soluciones habitacionales, lo cual indica que hay inicios de aprovechar este tipo de energía para la generación de electricidad.

Tabla 8

Irradiación Solar en Montecristi $\left(\mathrm{kWh} / \mathrm{m}^{2} / \mathrm{mes}\right)$

\begin{tabular}{lcccc}
\hline MES & METEONEORM & NASA & PVGIS TMY-SARAH & NREL NSRDB \\
\hline ENE & 142 & 170 & 106 & 131 \\
FEB & 132 & 148 & 113 & 125 \\
MAR & 163 & 180 & 160 & 148 \\
ABR & 168 & 172 & 172 & 152 \\
MAY & 157 & 162 & 133 & 141 \\
JUN & 117 & 123 & 142 & 123 \\
\hline
\end{tabular}


Tabla 8

Irradiación Solar en Montecristi ( $\left.\mathrm{kWh} / \mathrm{m}^{2} / m e s\right)$ (continuación)

\begin{tabular}{lcccc}
\hline MES & METEONEORM & NASA & PVGIS TMY-SARAH & NREL NSRDB \\
\hline JUL & 123 & 114 & 153 & 133 \\
AGO & 135 & 124 & 162 & 148 \\
2SEP & 127 & 131 & 152 & 147 \\
OCT & 105 & 136 & 150 & 148 \\
NOV & 117 & 138 & 151 & 147 \\
DIC & 144 & 162 & 152 & 150 \\
\hline ANUAL & 1,630 & 1,759 & 1,747 & 1,692
\end{tabular}

Los valores de irradiación solar para cada uno de los cantones muestran niveles aceptables de energía en los mismos, lo cual ratifica el potencial energético que tiene la provincia y la viabilidad técnica de instalar sistemas fotovoltaicos como fuente de generación de electricidad; sin embargo lo valores estimados son diferentes a los mostrados en el estudio del CONELEC respecto al potencial de energía fotovoltaica en la provincia, los cuales muestran un valor anual mayor por metro cuadrado en el año.

Adicionalmente, este trabajo de investigación ha empleado programas de simulación que permita evaluar el potencial teórico de energía eléctrica que se podría generar con un sistema fotovoltaico de autoconsumo o también denomina on-grid. Como variables de la simulación se ha considerado supuestos del sistema como un área total de paneles de $100.00 \mathrm{~m}^{2}$; luego se considera el valor promedio anual obtenido en cada cantón de la provincia e incorporar el efecto de paneles solares de silicio con su respectiva eficiencia para determinar el valor teórico de generación eléctrica que se puede entregar a la red.

Para cada simulación se ha considerado un sistema estándar básico de generación fotovoltaico, que emplea paneles solares, inversores, protectores, soportes de aluminio, cables solares y cajas para el manejo de la corriente que se genera. Como primera opción se estimó emplear paneles fotovoltaicos policristalinos de 60 celdas, que registran eficiencia entre $16 \%$ al $20 \%$; considerando que este tipo de panel tiene una alta demanda comercial en la actualidad. En la tabla 9 se puede apreciar el valor de generación promedio por cada cantón con una eficiencia estimada del panel de un $18 \%$. Se debe mencionar que en las simulaciones se mantiene la misma marca de inversor y resto de implementos para no generar distorsión en las pérdidas del sistema, tratando en todo momento que solo la variable panel fotovoltaico sea la que incida en la diferencia de la energía inyectada a la red. 


\section{Tabla 9}

Energía conectada a la red con paneles policristalinos $(k W h)$

\begin{tabular}{|c|c|c|c|c|c|c|}
\hline Generación de electricidad & PORTOVIEJO & MANTA & CHONE & EL CARMEN & JIPIJAPA & MONTECRISTI \\
\hline Irradiación global horizontal & $1,627.8$ & $1,791.8$ & $1,594.1$ & $1,401.1$ & $1,614.6$ & $1,706.9$ \\
\hline $\begin{array}{l}\text { Perdida por incidencia plano } \\
\text { receptor }\end{array}$ & $3.0 \%$ & $3.0 \%$ & $3.0 \%$ & $3.0 \%$ & $3.0 \%$ & $3.0 \%$ \\
\hline Perdida factor IAM en global & $2.5 \%$ & $2.5 \%$ & $2.5 \%$ & $2.5 \%$ & $2.5 \%$ & $2.5 \%$ \\
\hline Irradiación efectiva & $1,539.5$ & $1,694.5$ & $1,507.6$ & $1,325.1$ & $1,527.0$ & $1,614.3$ \\
\hline Conversión FV (KWh) & $27,710.9$ & $30,501.9$ & $27,137.6$ & $23,851.2$ & $27,485.7$ & $29,057.0$ \\
\hline Perdida de FV por irradiancia & $0.80 \%$ & $0.80 \%$ & $0.80 \%$ & $0.80 \%$ & $0.80 \%$ & $0.80 \%$ \\
\hline Perdida FV por temperatura & $8.00 \%$ & $8.00 \%$ & $8.00 \%$ & $8.00 \%$ & $8.00 \%$ & $8.00 \%$ \\
\hline Pérdida de calidad de modulo & $0.70 \%$ & $0.70 \%$ & $0.70 \%$ & $0.70 \%$ & $0.70 \%$ & $0.70 \%$ \\
\hline $\begin{array}{l}\text { Perdida de desajustes, } \\
\text { módulos y cadenas }\end{array}$ & $2.00 \%$ & $2.00 \%$ & $2.00 \%$ & $2.00 \%$ & $2.00 \%$ & $2.00 \%$ \\
\hline Perdida óhmica del cableado & $0.90 \%$ & $0.90 \%$ & $0.90 \%$ & $0.90 \%$ & $0.90 \%$ & $0.90 \%$ \\
\hline Energía del conjunto (KWh) & $24,274.7$ & $26,719.6$ & $23,772.5$ & $20,893.7$ & $24,077.5$ & $25,453.9$ \\
\hline $\begin{array}{l}\text { Perdida del inversor durante la } \\
\text { operación }\end{array}$ & $2.00 \%$ & $2.00 \%$ & $2.00 \%$ & $2.00 \%$ & $2.00 \%$ & $2.00 \%$ \\
\hline $\begin{array}{l}\text { Perdida en el inversor por el } \\
\text { umbral de potencia }\end{array}$ & $0.04 \%$ & $0.04 \%$ & $0.04 \%$ & $0.04 \%$ & $0.04 \%$ & $0.04 \%$ \\
\hline $\begin{array}{l}\text { ENERGIA INYECTADA A } \\
\text { LA RED (kWh) }\end{array}$ & $23,779.50$ & $26,174.50$ & $23,287.60$ & $20,467.40$ & $23,586.30$ & $24,934.7$ \\
\hline
\end{tabular}

Como un segundo escenario se ha considerado solo realizar cambios en el tipo de panel fotovoltaico, tomando para esta simulación un panel monocristalino, que normalmente alcanza a nivel de laboratorios una eficiencia mayor que los policristalinos entre $18 \%$ al $22 \%$. Para la simulación que se muestra en la tabla 10, se considera el valor promedio de generación anual de cada cantón y se estima la conversión con una eficiencia del panel del 20\%. Para ambos casos a pesar de que en los simuladores indican un acimut de cero grados y ángulos de inclinación entre $3^{0}$ y $10^{0}$; para alcanzar la posición optima de captación solar; se estimó en todos los casos con un ángulo de inclinación de $20^{0} \mathrm{y}$ acimut de $-150^{\circ}$, lo cual genera pérdidas por incidencia en el plano receptor. 
Tabla 10

Energía conectada a la red con paneles monocristalinos $(k W h)$

\begin{tabular}{|c|c|c|c|c|c|c|}
\hline $\begin{array}{l}\text { GENERACION DE } \\
\text { ELECTRICIDAD }\end{array}$ & PORTOVIEJO & MANTA & CHONE & EL CARMEN & JIPIJAPA & MONTECRISTI \\
\hline $\begin{array}{l}\text { IRRADIACION GLOBAL } \\
\text { HORIZONTAL }\end{array}$ & $1,627.8$ & $1,791.8$ & $1,594.1$ & $1,401.1$ & $1,614.6$ & $1,706.9$ \\
\hline $\begin{array}{l}\text { Pérdida por incidencia plano } \\
\text { receptor }\end{array}$ & $3.0 \%$ & $3.0 \%$ & $3.0 \%$ & $3.0 \%$ & $3.0 \%$ & $3.0 \%$ \\
\hline $\begin{array}{l}\text { Pérdida factor IAM en } \\
\text { global }\end{array}$ & $2.5 \%$ & $2.5 \%$ & $2.5 \%$ & $2.5 \%$ & $2.5 \%$ & $2.5 \%$ \\
\hline $\begin{array}{l}\text { IRRADIACION } \\
\text { EFECTIVA }\end{array}$ & $1,539.5$ & $1,694.5$ & $1,507.6$ & $1,325.1$ & $1,527.0$ & $1,614.3$ \\
\hline Conversión FV (KWh) & $30,789.8$ & $33,891.0$ & $30,152.9$ & $26,501.3$ & $30,539.7$ & $32,285.5$ \\
\hline $\begin{array}{l}\text { Pérdida de FV por } \\
\text { irradiancia }\end{array}$ & $0.80 \%$ & $0.80 \%$ & $0.80 \%$ & $0.80 \%$ & $0.80 \%$ & $0.80 \%$ \\
\hline Perdida FV por temperatura & $8.00 \%$ & $8.00 \%$ & $8.00 \%$ & $8.00 \%$ & $8.00 \%$ & $8.00 \%$ \\
\hline $\begin{array}{l}\text { Pérdida de calidad de } \\
\text { modulo }\end{array}$ & $0.70 \%$ & $0.70 \%$ & $0.70 \%$ & $0.70 \%$ & $0.70 \%$ & $0.70 \%$ \\
\hline $\begin{array}{l}\text { Pérdida de desajustes, } \\
\text { módulos y cadenas }\end{array}$ & $2.00 \%$ & $2.00 \%$ & $2.00 \%$ & $2.00 \%$ & $2.00 \%$ & $2.00 \%$ \\
\hline Pérdida óhmica del cableado & $0.90 \%$ & $0.90 \%$ & $0.90 \%$ & $0.90 \%$ & $0.90 \%$ & $0.90 \%$ \\
\hline $\begin{array}{l}\text { ENERGIA DEL } \\
\text { CONJUNTO (KWh) }\end{array}$ & $26,971.9$ & $29,688.5$ & $26,413.9$ & $23,215.2$ & $26,752.8$ & $28,282.1$ \\
\hline $\begin{array}{l}\text { Perdida del inversor durante } \\
\text { la operación }\end{array}$ & $2.00 \%$ & $2.00 \%$ & $2.00 \%$ & $2.00 \%$ & $2.00 \%$ & $2.00 \%$ \\
\hline $\begin{array}{l}\text { Perdida en el inversor por el } \\
\text { umbral de potencia }\end{array}$ & $0.04 \%$ & $0.04 \%$ & $0.04 \%$ & $0.04 \%$ & $0.04 \%$ & $0.04 \%$ \\
\hline $\begin{array}{l}\text { ENERGIA INYECTADA } \\
\text { A LA RED (kWh) }\end{array}$ & $26,421.67$ & $29,082.83$ & $25,875.07$ & $22,741.58$ & $26,207.01$ & $27,705.18$ \\
\hline
\end{tabular}

Como un último escenario se ha considerado un sistema con paneles de silicio de tipo amorfo, los cuales se caracterizan por su bajo costo y poca eficiencia de conversión energética, los mismos que alcanzan eficiencias teóricas entre un $10 \%$ al $14 \%$. Considerando para la simulación una eficiencia del 12\%; y manteniéndose el porcentaje de perdida en el inversor al convertir la corriente continua a corriente alterna, las perdidas por el tipo de cable estimado y otras perdidas que se muestran en la tabla 11. 


\section{Tabla 11}

Energía conectada a la red con paneles de silicio amorfo ( $k W h)$

\begin{tabular}{|c|c|c|c|c|c|c|}
\hline $\begin{array}{l}\text { GENERACION DE } \\
\text { ELECTRICIDAD }\end{array}$ & PORTOVIEJO & MANTA & CHONE & EL CARMEN & JIPIJAPA & MONTECRISTI \\
\hline $\begin{array}{l}\text { IRRADIACION GLOBAL } \\
\text { HORIZONTAL }\end{array}$ & $1,627.8$ & $1,791.8$ & $1,594.1$ & $1,401.1$ & $1,614.6$ & $1,706.9$ \\
\hline $\begin{array}{l}\text { Perdida por incidencia plano } \\
\text { receptor }\end{array}$ & $3.0 \%$ & $3.0 \%$ & $3.0 \%$ & $3.0 \%$ & $3.0 \%$ & $3.0 \%$ \\
\hline $\begin{array}{l}\text { Perdida factor IAM en } \\
\text { global }\end{array}$ & $2.5 \%$ & $2.5 \%$ & $2.5 \%$ & $2.5 \%$ & $2.5 \%$ & $2.5 \%$ \\
\hline $\begin{array}{l}\text { IRRADIACION } \\
\text { EFECTIVA }\end{array}$ & $1,539.5$ & $1,694.5$ & $1,507.6$ & $1,325.1$ & $1,527.0$ & $1,614.3$ \\
\hline Conversión FV (KWh) & $18,473.9$ & $20,334.6$ & $18,091.7$ & $15,900.8$ & $18,323.8$ & $19,371.3$ \\
\hline $\begin{array}{l}\text { Perdida de FV por } \\
\text { irradiancia }\end{array}$ & $0.80 \%$ & $0.80 \%$ & $0.80 \%$ & $0.80 \%$ & $0.80 \%$ & $0.80 \%$ \\
\hline Perdida FV por temperatura & $8.00 \%$ & $8.00 \%$ & $8.00 \%$ & $8.00 \%$ & $8.00 \%$ & $8.00 \%$ \\
\hline $\begin{array}{l}\text { Pérdida de calidad de } \\
\text { modulo }\end{array}$ & $0.70 \%$ & $0.70 \%$ & $0.70 \%$ & $0.70 \%$ & $0.70 \%$ & $0.70 \%$ \\
\hline $\begin{array}{l}\text { Perdida de desajustes, } \\
\text { módulos y cadenas }\end{array}$ & $2.00 \%$ & $2.00 \%$ & $2.00 \%$ & $2.00 \%$ & $2.00 \%$ & $2.00 \%$ \\
\hline Perdida óhmica del cableado & $0.90 \%$ & $0.90 \%$ & $0.90 \%$ & $0.90 \%$ & $0.90 \%$ & $0.90 \%$ \\
\hline $\begin{array}{l}\text { ENERGIA DEL } \\
\text { CONJUNTO (KWh) }\end{array}$ & $16,183.1$ & $17,813.1$ & $15,848.4$ & $13,929.1$ & $16,051.7$ & $16,969.3$ \\
\hline $\begin{array}{l}\text { Perdida del inversor durante } \\
\text { la operación }\end{array}$ & $2.00 \%$ & $2.00 \%$ & $2.00 \%$ & $2.00 \%$ & $2.00 \%$ & $2.00 \%$ \\
\hline $\begin{array}{l}\text { Perdida en el inversor por el } \\
\text { umbral de potencia }\end{array}$ & $0.04 \%$ & $0.04 \%$ & $0.04 \%$ & $0.04 \%$ & $0.04 \%$ & $0.04 \%$ \\
\hline $\begin{array}{l}\text { ENERGIA INYECTADA } \\
\text { A LA RED }(\mathbf{k W h})\end{array}$ & $15,853.00$ & $17,449.70$ & $15,525.04$ & $13,644.95$ & $15,724.21$ & $16,623.11$ \\
\hline
\end{tabular}

\section{Conclusiones}

- En la presente investigación se analiza el potencial energético solar en la provincia de Manabí, como una opción de fuente primaria para la generación de electricidad a nivel comercial, habitacional y empresarial. Este trabajo de investigación plantea que esta fuente de energía puede contribuir al cambio de la matriz energética que busca el país, de acuerdo con las políticas y acciones 
planificadas para el cumplimento de los objetivos de desarrollo sostenible. La energía solar fotovoltaica muestra un desarrollo tecnológico que hace viable su instalación en la provincia de Manabí, lo cual coincide con estudios realizados en países de la región. Los casos tomados en las principales ciudades de Manabí indican el alto potencial energético de Manta, Montecristi y Portoviejo; aunque los niveles alcanzados en las otras ciudades indican que una inversión en este tipo de energía puede ser viable desde el punto de vista financiero. Estos resultados preliminares también indican la importancia de la decisión administrativa de empresas sobre la opción de invertir en sistemas fotovoltaicos, los mismos que generan beneficios al ambiente en la generación de electricidad, $\mathrm{y}$ que requieren de acciones y políticas empresarial orientadas a la responsabilidad social corporativa, distribuyendo recursos y conocimiento para beneficio de los grupos de interés de las organizaciones. La generación de electricidad por medio de paneles fotovoltaicos de Silicio se observa viables desde el punto de vista técnico, apreciándose los valores de energía inyectada a la red que podría generar estos sistemas para beneficio de las organizaciones o familias. Por último, se considera que al existir sectores de la provincia que por su geográfica no logran tener acceso a electricidad, este tipo de sistema es una opción viable y segura para proveer de este servicio básico a pequeños y medianos negocios situados en esta región, más aún sectores como el agrícola, pecuario y artesanal que en muchos de los casos no pueden contar con electricidad para el desarrollo de sus procesos productivos, generando afectación a su crecimiento económico y de calidad de vida.

\section{Limitaciones}

Esta investigación no es un estudio definitivo sobre el potencial de la irradiación solar en la provincia de Manabí y su viabilidad técnica para instalar estos sistemas para la generación de electricidad. El presente trabajo plantea la posibilidad que en la provincia se puede instalar sistemas fotovoltaicos para la generación de energía, que puede y debe ser aprovechada por diferentes agentes económicos que tienen limitaciones por su falta de acceso al sistema público de electricidad. Parte de las limitaciones de este trabajo es que solo se tomó un punto de cada cantón, lo cual debe ampliarse para poder generalizar los resultados y de esta manera determinar una viabilidad técnica. De igual manera, a pesar de considerar varias bases de datos, los valores muestran diferencias altas entre las simulaciones, esto indica que se requiere para una mayor generalización de resultados, tomar valores de instalaciones reales situadas en dichos cantones que permitan una mejor estimación energética de la provincia. Así mismo, solo se ha considera la viabilidad técnica de la generación de electricidad por medio de un sistema fotovoltaico, sin analizar desde el punto de vista económico el retorno financiero en la inversión de este tipo de sistema; el cual puede requerir fuentes de financiamiento no tradicionales 


\section{www.cienciadigital.org}

como el crowdfunding (Rugel et al., 2017), tradicionales como el sistema financiero y mercado de valores.

\section{Referencias Bibliográficas}

Alcántara, V., \& Padilla, E. (2005). Análisis de las emisiones de CO2 y sus factores explicativos en las diferentes áreas del mundo. Revista de Economía Crítica, 4, 17-37.

Agencia de Regulación y Control de Electricidad [ARCONEL]. (2019). Atlas del Sector Eléctrico Ecuatoriano 2018. Quito:

Asamblea General. (2015). Transformar nuestro mundo: la Agenda 2030 para el Desarrollo Sostenible. Ginebra: Organización de las Naciones Unidas.

Ayuso, S. (2020). ¿Cómo contabilizar el impacto ambiental de las empresas? El caso de las emisiones de gases de efecto invernadero. Revista de Estudios Empresariales, 94-111.

Bórquez, B., \& Lopicich, B. (2017). La dimensión bioética de los Objetivos de Desarrollo Sostenible (ODS). Revista de bioética y derecho, 41, 121-139.

Brandão, M., Kirschbaum, M., Cowie, A., \& Vedel, S. (2019). Quantifying the climate change effects of bioenergy systems: Comparison of 15 impact assessment methods. GCB Bioenergy, 11(5), 727-743.

Canaza-Choque, F., \& Huanca-Arohuanca, J. (2018). Perú 2018: hacia una Educación Intercultural Bilingüe sentipensante. Sciéndo. Ciencia Para El Desarrollo, 21(4), 515-522.

Caraballo Pou, M., \& García-Simón, J. (2017). Energías renovables y desarrollo económico. Un análisis para España y las grandes economías europeas. El trimestre económico, 84(335), 571-609.

Dong, Y., Hauschild, M., Sørup, H., Rousselet, R., \& Fantke, P. (2019). Evaluating the monetary values of greenhouse gases emissions in life cycle impact assessment. Journal of Cleaner Production, 209, 538-549.

Feldman, D., \& Margolis, R. (2021). H2 2020: Solar Industry Update. Abu Dabi: NREL.

Feldman, D., Wu, K., \& Margolis, R. (2021). Solar Industry Update. Golden: NREL. 


\section{www.cienciadigital.org}

García, C., López, J., \& Gómez, T. (2021). Estimación del costo de distribución de la energía eléctrica en Colombia considerando generación distribuida fotovoltaica. Información tecnológica, 32(1), 79-88.

Gielen, D., Boshell, F., Saygin, D., Bazilian, M., Wagner, N., \& Gorini, R. (2019). The role of renewable energy in the global energy transformation. Energy Strategy Reviews, 24, 38-50.

Gil, C. (2018). Objetivos de Desarrollo Sostenible (ODS): una revisión crítica. Papeles de relaciones ecosociales y cambio global, 140, 107-118.

Gómez, C. (2018). Objetivos de Desarrollo Sostenible (ODS): una revisión crítica. Papeles de relaciones ecosociales y cambio global, 140, 107-118.

González, E., \& Meira, P. (2020). Educación para el cambio climático: ¿Educar sobre el clima o para el cambio? Perfiles educativos, 42(168), 157-174.

Harjanne, A., \& Korhonen., J. (2019). Abandoning the concept of renewable energy. Energy policy, 127, 330-340.

Instituto Nacional de Estadísticas y Censos [INEC]. (2010). Censo de población y vivienda del Ecuador 2010. Quito: Inec.

IPCC. (2020). El cambio climático y la tierra. Ginebra: Grupo Intergubernamental de Expertos sobre el Cambio Climático.

IRENA. (2021). Renewable capacity statistics 2021. Abu Dabi: IRENA.

Lewandowsky, S., Oreskes, N., Risbey, J., Newell, B., \& Smithson, M. (2015). Seepage: Climate change denial and its effect on the scientific community. Global Environmental Change, 33, 1-13.

Monteiro, N., Da silva, E., \& Moita, J. (2019). Sustainable development goals in mining.". Journal of Cleaner Production, 228, 509-520.

Pérez, R., \& Osal, W. (2018). Gases de efecto invernadero por generación de electricidad en. Publicaciones en Ciencias y Tecnología, 13(1), 30-40.

Perpiñán, O. (2013). Energía solar fotovoltaica. Madrid: Creative Commons ebook.

Poma, A. (2018). El papel de las emociones en la respuesta al cambio climático. Inter disciplina, 6(15), 191-214. 
Reid, A., Brooks, J., Dolgova, L., Laurich, B., Sullivan, B., Szekeres, P., et al. (2017). Post-2015 Sustainable Development Goals still neglecting their environmental roots in the Anthropocene. Environmental Science and Policy, 179-184.

Rugel, C., González, F., Navas, W., Torres, P., \& Vera, D. (2017). CROWDFUNDING: una alternativa de financiamiento para las PYMES. Guayaquil: Grupo Compas.

Sachs, J., Schmidt-Traub, G., Mazzucato, M., Messner, D., Nakicenovic, N., \& Rockström, J. (2019). Six transformations to achieve the sustainable development goals. Nature Sustainability, 2, 805-814.

Schewe, J., Gosling, S., Reyer, C., Zhao, F., \& Ciais, P. (2019). Jacob, Simon Gosling, Christopher Reyer, Fang Zhao, Philippe Ciais y Josua Elliott. Nature Communications, 10, 1-14.

Vera, C., Navas, W., \& Amén, C. (2017). Algunas especificaciones acerca de la administración de empresas. Dominio de las ciencias, 284-298.

Wang, H., Lei, Z., Zhang, X., Zhou, B., \& Peng, J. (2019). A review of deep learning for renewable energy forecasting. Energy Conversion and Management, 1-16.

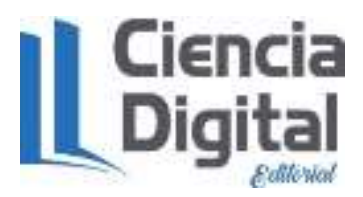


El artículo que se publica es de exclusiva responsabilidad de los autores y no necesariamente reflejan el pensamiento de la Revista Ciencia Digital.

\section{LCiencia}

El artículo queda en propiedad de la revista y, por tanto, su publicación parcial y/o total en otro medio tiene que ser autorizado por el director de la Revista Ciencia Digital.

\section{latinde catálogo}
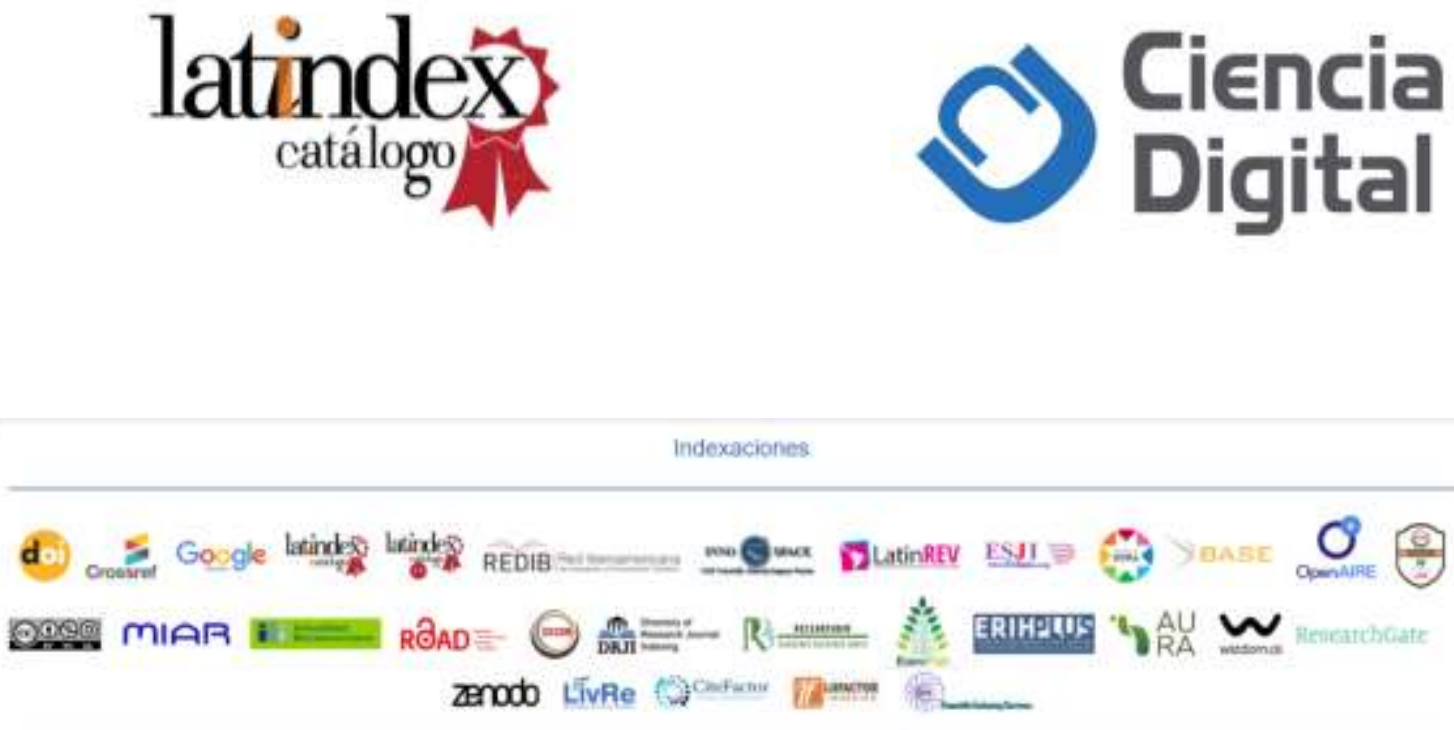